\title{
El Progreso de las Tecnología de Información y Comunicación en el Ámbito Educativo
}

Alizia Agnelli Faggioli.

Universidad Metropolitana. Quito. Ecuador.

Autor para la correspondencia: aliziagnelli@gmail.com

ORCID: http://orcid.org/0000-0003-3775-8977

Fecha de recepción: 10 de enero de 2020

Fecha de aceptación: 05 de abril de 2020

\section{Resumen}

El presente trabajo tuvo como objetivo, analizar a las tecnologías de información y comunicación en la educación en el escenario de transformaciones, debido a que la enseñanza y el proceso educativo deben machar a la par de los cambios que se viven a diario en el contexto local, regional, nacional, internacional y global, donde el desarrollo del internet y las tecnología de información y comunicación, han creado en las personas y en la sociedad en general un conocimiento en la forma de vivir el presente y de plantearse en el futuro grandes cambios. Su alcance consiste en la necesidad de entender que los ambientes telemáticos han permitido una interacción de comunicaciones a través de una cadena de redes que ha logrado que el proceso de enseñanza-aprendizaje sea de una forma efectiva. Desde la perspectiva metodológica la investigación fue documental a través del análisis de las fuentes bibliográficas sobre la temática. Los resultados permitieron determinar que este sistema que abarca educadores y alumnos, les posibilita interactuar en un tiempo real. Se concluyó que a través de las tecnologías de información y comunicación se ha logrado una evolución en la educación porque ha reformado la manera de enseñar y donde el rol del docente y el estudiante juegan un papel importante.

Palabras Clave: Tecnología. Información, Comunicación, Educación. Abstract

The objective of this work was to analyze the information and communication technologies in education in the scenario of transformations, because teaching and the educational process must kill along with the changes that are experienced daily in the local context. , regional, national, international and global, where the development of the internet and information and communication technology, have created in people and in society in general a knowledge in the way of living in the present and in the future considering major changes. Its scope consists in the need to understand that telematic environments have allowed a communication interaction through a chain of networks that has made the teaching-learning process effective. From the methodological perspective the research was documentary through the analysis of bibliographic sources on the subject. The results allowed us to determine that this system, which includes educators and students, allows them to interact in real time. It was concluded that through the 
information and communication technologies an evolution in education has been achieved because it has reformed the way of teaching and where the role of the teacher and the student play an important role.

Keywords: Technology. Information. Communication. Education.

\section{Introducción}

La educación se encuentra en un tiempo de transformaciones que requiere desafíos y requerimientos, específicamente en la construcción del conocimiento que plantea el adelanto de capacidades y competencias profesionales, que faciliten que los individuos en el contenido de la sociedad del conocimiento sean competentes de posicionarse e integrarse en ella, lo que en consecuencia constituye el fundamento del proceso educativo.

Las sociedades son fruto de sus actividades económicas, sociales, educativas, políticas y medioambientales, las cuales están en constante movimiento y relacionadas entre sí, en especial en un momento donde la rapidez de la comunicación se ha hecho evidente producto de la competitividad. Y precisamente sobre esa competitividad se exige la innovación de conocimientos para los que se va a insertar en el mundo educativo, donde se requiere de un pensamiento transformador, crítico, valioso e integral, aplicado a este contexto.

Belandro (2015) afirma que "la educación, es un proceso que se fundamenta en la obtención de conocimientos, habilidades, valores y hábitos en un grupo humano" (pág.46). Se considera una institución al servicio del hombre, de su progreso y aprendizaje, en el que adquiere y se ilustra de conocimientos, y donde las tecnologías de la información y la comunicación, logran complementar, enriquecer y transformar la educación.

Las tecnologías de información y comunicación denominadas TICs, según Díaz (2017) la define como "el conjunto de tecnologías que permiten la adquisición, producción, almacenamiento, tratamiento, transmisión, registro y presentación de información en forma de voz, imágenes y datos contenidos en señales de naturaleza acústica, óptica o electromagnética" (p.65). La incorporación de las TICs en el ámbito de la educación es una realidad y necesidad, por lo que nadie puede pensar en que estos medios de divulgación y facilitación del acceso a la información puedan estar fuera del hecho educativo, porque proporciona un aprendizaje constructivo y significativo.

En base a lo anterior el propósito de este tema es que el estudiante funde su saber con la unión de los conocimientos que tiene con la obtención de nuevas informaciones y de esta manera aprende por medio de ella a través de la búsqueda de nuevas tecnologías. La justificación e importancia se debe a que el impacto y huella de las tecnologías de información y comunicación en el ámbito educativo a cualquier nivel, viene a ser una salida alternativa al proceso de enseñanza-

Esta obra se comparte bajo la licencia Creative Common Atribución-No Comercial 4.0 International (CC BY-NC 4.0) 
aprendizaje habitual, en virtud que contribuye a generar cambios por su constante evolución, por lo que requiere que el docente y el alumno vayan adaptándose también para asegurar que, con el uso de los equipos digitales ese proceso sea seguro y favorable.

Todo ello es debido a como expresa Belloch (2012) "Las Tecnologías de la Información y la Comunicación, son todas aquellas que giran en torno a las tecnologías de almacenamiento, procesamiento, recuperación y comunicación de la información a través de diferentes dispositivos electrónicos e informáticos" (p.97), por lo que requiere que el docente y el alumno vayan adaptándose también para asegurar que, con el uso de los equipos digitales ese proceso sea seguro y favorable.

Sin embargo, en la actualidad el problema sobre las TICs surge por el desconocimiento del uso de ellas por parte de los docentes que no se adaptan a las nuevas herramientas tecnológicas o porque no se cuenta con la tecnología adecuadas dentro de las instituciones educativas. Por ello, el uso de ellas, surge como una necesidad en los actuales momentos, donde se debe desarrollar la creatividad, así como la formación para utilizar y promover ambientes de trabajo participativos, significativos, dinámicos y flexibles, en virtud que ante el rápido y exponencial crecimiento cultural y social de siglo XXI, las normas que regulan el comportamiento humano, en cualquiera de sus manifestaciones, requieren de adaptación rápida y constante, pues de no hacerlo corren el riesgo del desuso.

\section{Materiales y Métodos}

La investigación es de tipo teórica en tanto sistematiza los sustentos teóricos sobre la Tecnología de Información y Comunicación en el Ámbito Educativo. La información teórica se recopila con la indagación que sobre el tema se realizó en textos, páginas web, artículos científicos.

Se emplearon métodos teóricos como análisis y síntesis, inducción y deducción de lo abstracto a lo concreto, que se utilizan en el estudio de la bibliografía consultada, con el fin de sistematizar sustentos teóricos sobre la Tecnología de Información y Comunicación en el Ámbito Educativo.

\section{Resultados y Discusión}

\section{Las tecnologías de información y comunicación en el ámbito educativo.}

La presente sociedad de la información y tecnología, con su extraordinaria expansión, ha originado cambios en la apreciación de los diferentes actores de las instituciones sociales que tenían respecto de la educación sustentada en la Web y las TICs. Sin embargo, este cambio de

Esta obra se comparte bajo la licencia Creative Common Atribución-No Comercial 4.0 International (CC BY-NC 4.0) 
modelo no ha sido un proceso fácil.

Sus inicios son a mediados del siglo XX donde las múltiples tentativas institucionales que nacieron para generar un posicionamiento de las TICs en la educación en el contexto Latinoamericano chocaron con la muralla de los gobiernos de esos países, a pesar que algunos se mostraron contrarios a la justificación de la modalidad, para aprobar, regular y evaluar esos programas. Sin embargo, no fue sino hasta la aparición de la Web en los años noventa, conjuntamente con las tecnologías asociadas que se fueron desarrollando, donde los gobiernos latinoamericanos fueron cediendo al cambio y apropiándose de la versatilidad que otorga el manejo inmediato de la información y la facilidad para divulgarla.

Este crecimiento acentuado de conectividad a la Web de los pueblos Latinoamericanos y la influencia ineludible de las TICs en el progreso económico regional, obligaron a que los gobiernos regionales Latinoamericanos asumieran una actitud positiva respecto de estas y la conveniencia de incorporarlas en la educación como un apoyo para propiciar el desarrollo económico soportado en la misma educación.

Por otra parte, es necesario entender que las TICs no son un fin en sí mismas, en virtud que la Web y toda la tecnología desarrollada y sustentada en esta, constituyen un medio, un instrumento, una plataforma para el desarrollo. No depende de éstas lograr o generar el fenómeno de la evolución social y humana, no puedan por sí solas determinar el avance de las sociedades y mejorar su calidad de vida, en virtud que se requiere de la acción decidida y coherente de los gobernantes y de los demás actores institucionales para lograr, con el apoyo de las TICs, disminuir las diferencias sociales.

Belloch (2012) expresa. "Las Tecnologías de la Información y la Comunicación, son todas aquellas que giran en torno a las tecnologías de almacenamiento, procesamiento, recuperación y comunicación de la información a través de diferentes dispositivos electrónicos e informáticos" (p.105). Ahora bien, siendo que la educación está llamada a ser la piedra angular del desarrollo humano individual y colectivo, tiene un rol protagónico para garantizar una evolución social en forma sostenible, todo ello, porque la educación debe ser el elemento más permeable y adaptable a los cambios y las necesidades humanas.

Las tecnologías de la información y la comunicación, tienen influencia en la manera de entender y ejecutar el hecho educativo, han incursionado en sus niveles y modalidades y han creado el espacio virtual para llevar la información a todos los espacios de la geografía del planeta, contribuyendo a la construcción y creación de más conocimiento en solo una década, del que se

Esta obra se comparte bajo la licencia Creative Common Atribución-No Comercial 4.0 International (CC BY-NC 4.0)

Revista Trimestral del Instituto Superior Universitario Espíritu Santo 
ha producido en los doscientos años anteriores.

La educación en la diversidad de sus niveles, está ahora a la par de alcanzar la información más reciente, el hecho más influyente en el quehacer humano y el conocimiento más actualizado, todo para poder garantizar una educación más competitiva en un contexto y un mercado laboral altamente excluyente para aquellos que no manejan la información. Por lo tanto, las instituciones deben realizar la inversión en tecnologías de la información y comunicación, a través de un adiestramiento para el uso correcto de las mismas, debido a que no puede existir calidad en un organismo educativo si no hay una apropiada gestión de recursos tecnológicos y su creciente unificación en los diferentes espacios curriculares, en ese sentido, la utilización de las TICs, va más allá del aula, favoreciendo las nuevas formas de aprendizaje y contribuyendo a la búsqueda de más recursos para lograr que el estudiante tenga atención a la variedad.

Todo esto, porque las TICs pueden proporcionar a ciertos alumnos con algunas dificultades, un camino rápido al aprendizaje provocando la independencia de estos frente a los trabajos diarias que surjan en el aula, lo que se traduce en igualdad y equidad en base al acceso del conocimiento a todos los educandos.

\section{Progreso de las tecnologías de información y comunicación}

El avance y desarrollo que ha tenido las TICs, es producto del nuevo enfoque del conocimiento y aprendizaje, según lo expresan Soto, Serna y Neira (2017), "han cambiado las posiciones que se han desempeñado en las instituciones educativas y en los procesos de enseñanza-aprendizaje, igual que a la creación y difusión del conocimiento en las estrategias y políticas públicas fijadas en las actuales expectaciones curriculares" (p.53). De ahí que el proceso de la enseñanza ha contribuido a varios progresos manifestados en calidad, conectividad, elasticidad, administración de tiempos, áreas para la enseñanza, el razonamiento, solucionar indecisiones y lograr información de forma interactiva, en línea.

La incorporación de las TICs, extiende un abanico de posibilidades de ofertas educativas a nivel mundial, que admite varias opciones en el lugar interactivo, nivel de conocimiento y el grado de formación que requiere y desea alcanzar el estudiante. Con ello, se accede a la información, a través de la red, trabajar en equipo, realizar trabajos, investigaciones, conservar la información de manera organizada y actualizada de forma permanente con mayor eficacia.

Uno de los progreso de las tecnologías de información y comunicación de mayor transcendencia ha sido la educación virtual, lo cual se ha redefinido y redimensionado gracias a la Web y a las tecnologías asociadas, que han traído consigo un sinfín de oportunidades, solo limitadas por la creatividad del hombre y la gestión de los gobiernos. Los nuevos procesos tecnológicos pueden proveer los mecanismos para el progreso de los procesos de enseñanza y del

Esta obra se comparte bajo la licencia Creative Common Atribución-No Comercial 4.0 International (CC BY-NC 4.0)

Revista Trimestral del Instituto Superior Universitario Espíritu Santo 
aprendizaje y para la tarea de los ambientes educativos. Sin embargo, afirma Tedesco (2005), "no es que se quiera negar la potencialidad e innovación de las tecnologías, sino enfatizar que el ejercicio de ella no estriba de las tecnologías mismas sino de los modelos pedagógicos que se manejen". (p.92). Este autor sostiene que la disminución de las diferencias sociales no se origina de las TICs, sino de las políticas educativas que puedan ser aplicadas por los gobiernos y gracias a las cuales logren insertarse, siendo su apreciación acertada toda vez que el éxito o fracaso de las TICs en la educación dependerá en gran medida del verdadero interés de los docentes, instituciones educativas y gobiernos en lograr su inclusión efectiva en el ámbito educativo.

\section{Las transformaciones de las tecnologías de información y comunicación en el ámbito educativo}

Las TICs integran los cambios desde todos los ámbitos que se están originando en la actualidad, y los organismos de educación no pueden estar alejados de esa realidad. Es necesario que se adapten a las particularidades individuales y a lo que requiere el alumnado, para facilitar su trayectoria académica y desarrollar sus potencialidades. Durante el proceso de enseñanzaaprendizaje, el estudiante necesita de la continuidad de escenarios de carácter determinado que ayuda al aprovechamiento de conocimientos en la ejecución y progreso de las distintas labores. Cada estudiante tiene una capacidad individualizada para la adquisición de nuevos conocimientos y su ritmo de aprendizaje será acorde a la asimilación y adquisición de los mismos.

La justificación de los entornos que deben alcanzar para certificar que con la utilización de los equipos digitales en las distintas tareas que debe realizar, se va construyendo para que el proceso de enseñanza-aprendizaje sea propicio, a través del uso de recursos tecnológicos que benefician la actividad en el interior de las aulas y fuera de ellas, donde los estudiantes y docentes comparten e intercambian información en tiempo real como son: Google Apps for education, Edmodo, Moodle, GoConqr, Padlet, Co, Glogster, Popplet. A su vez están las distintas herramientas en línea como Dropbox, Google Drive, y como juegos interactivos que sirven en el proceso educativo, están Kahoot. Todo lo anterior es de vital importancia, debido a que estos recursos de tecnología tienen y cumplen un nuevo desempeño para los profesores y los estudiantes, lo cual se funda en la adquisición de un aprendizaje donde se favorece del sentido crítico y las estrategias para la adquisición de los saberes.

Los espacios virtuales se están convirtiendo en el nuevo lenguaje donde todos interactúan, para ello, es importante que los docentes tengan una cierta preparación sobre el uso y conocimiento de los métodos y técnicas de las TICs para brindar una educación de eficacia a todos los estudiantes, en virtud que la educación requiere ser pensada desde una nueva visión, de complementariedad, lo que requiere además de reconocer la necesidad de incorporar al sistema

Esta obra se comparte bajo la licencia Creative Common Atribución-No Comercial 4.0 International (CC BY-NC 4.0)

Revista Trimestral del Instituto Superior Universitario Espíritu Santo 
educativo el manejo de las innovaciones tecnológicas.

La educación, concebida como mecanismos de creación de conocimientos y formadores de profesionales que serán garantes del manejo de los destinos de la sociedad, están llamadas a reflexionar sus lazos con la sociedad productiva, a evaluar cómo se están llevando a cabo los procesos internos y a programar una nueva conducción desde el mismo seno de las instituciones educativas, en el uso de las TICs para el reajuste y formación de hombres y mujeres, que actúen oportunamente, eficientemente y efectivamente a las necesidades de la ciudadanía propendiendo a un afianzamiento del adelanto económico que los países exigen.

Por otra parte, las instituciones educativas, deben propiciar y promover las ideas para la generación de una regulación normativa que auspicie el desarrollo de un nuevo enfoque educativo, efectivo y flexible, que se fortalezca en el uso de las TICs y de esta manera suministrar a los estudiantes, la preparación necesarios para este siglo. Esta colectividad en formación, solo recogerá su efectivo sentido, si se convierte en un medio al servicio de la construcción a nivel mundial de sociedades del conocimiento que sean fuentes de desarrollo para todos y, sobre todo, para los países menos adelantados.

\section{Conclusiones}

La influencia que en la vida cotidiana de todos los seres humanos tienen hoy las tecnologías de la información y la comunicación en todos los ámbitos del quehacer humano han venido a afectar nuevas y más amplias visiones del pensamiento humano y en especial en el ámbito educativo donde el desarrollo exponencial de la tecnología ha tenido un crecimiento en los últimos diez años.

Las TICs, pueden ayudar a los docentes a fundar una sociedad con conocimiento global, porque admiten desarrollar capacidades de invención que pueden ser definitivos en el adelanto de la sociedad y que inciden sobre el desarrollo sostenible a nivel global, por ello, la necesidad y la obligación para las instituciones educativas de garantizar una Educación del nivel que requiere un mercado de trabajo cada día más competitivo, interdependiente y globalizado.

Los diferentes inconvenientes que pudiera tener las TICs, debe ser superados en armonía entre la institución educativa, los docentes y la esfera familiar, debido a la importancia del uso de herramientas informáticas en el contexto educativo, lo que se hace necesario que las planificación desde las aulas se observen estos hechos, de manera que envuelvan actividades que se funden en la utilización de estas herramientas, fomentando de esta manera una metodología más conforme

Esta obra se comparte bajo la licencia Creative Common Atribución-No Comercial 4.0 International (CC BY-NC 4.0) 
con la elaboración académica que la sociedad requiere a los futuros profesionales que en estos momentos se está formando.

\section{Referencias Bibliográficas}

Belandro, M. M.(2015). La Educación Repensada. Madrid. España: Ediciones Pirámide.

Belloch, C. O. (2012). Las Tecnologías de la Información y Comunicación en el aprendizaje. Madrid. Espana: Universidad de Valencia. Recuperado de http://www.uv.es/bellochc/pedagogia/EVA1.

Díaz, R. (2017). La Gestión de la Innovación en la práctica educativa con las TIC: Escuela de Educación. Caracas. Venezuela: Editorial Universidad Central de Venezuela.

Soto, C. Serna, A, y Neira, M. (2017). Ventajas del uso de las TICs en el Proceso de enseñanza-aprendizaje desde la óptica de los Docentes Universitarios Españoles. Madrid. España: Recuperado de edutec.rediris.es/revelec2/revelec.

Tedesco, J. (2005). Las TICs y la Desigualdad Educativa en América Latina. Tercer Seminario: Las Tecnologías de Información y Comunicación y los Desafios del Aprendizaje en la Sociedad del Conocimiento. 30 de marzo y el 1 de abril de 2005. Santiago de Chile. Chile: Seminario CEDI/OCDE de Habla Hispana.

de Habla Hispana.

Esta obra se comparte bajo la licencia Creative Common Atribución-No Comercial 4.0 International (CC BY-NC 4.0) 